\title{
Shortcomings of the Commercial MALDI-TOF MS Database and Use of MLSA as an Arbiter in the Identification of Nocardia Species
}

\author{
Gema Carrasco ${ }^{1}$, Juan de Dios Caballero², Noelia Garrido', Sylvia Valdezate ${ }^{1 *}$, \\ Rafael Cantón ${ }^{2}$ and Juan A. Sáez-Nieto ${ }^{1}$ \\ 'Área de Bacteriología, Centro Nacional de Microbiología, Instituto de Salud Carlos III, Madrid, Spain, ${ }^{2}$ Servicio de \\ Microbiología, Hospital Universitario Ramón y Cajal and Instituto Ramón y Cajal de Investigación Sanitaria, Madrid, Spain
}

\section{OPEN ACCESS}

Edited by:

David W. Graham,

Newcastle University, UK

Reviewed by:

Ying Zhou,

AmberGen Inc., USA

Marcos Quintela-Baluja,

Newcastle University, UK

*Correspondence:

Sylvia Valdezate

svaldezate@isciii.es

Specialty section: This article was submitted to Antimicrobials, Resistance

and Chemotherapy,

a section of the journal Frontiers in Microbiology

Received: 26 November 2015 Accepted: 04 April 2016 Published: 21 April 2016

Citation:

Carrasco G, de Dios Caballero J, Garrido N, Valdezate S, Cantón R and Sáez-Nieto JA (2016)

Shortcomings of the Commercial MALDI-TOF MS Database and Use of MLSA as an Arbiter in the Identification of Nocardia Species. Front. Microbiol. 7:542. doi: 10.3389/fmicb.2016.00542
Nocardia species are difficult to identify, a consequence of the ever increasing number of species known and their homogeneous genetic characteristics. 16S rRNA analysis has been the gold standard for identifying these organisms, but proteomic techniques such as matrix-assisted laser desorption ionization-time of flight (MALDI-TOF MS) and housekeeping gene analysis, have also been explored. One hundred high $(n=25)$, intermediate $(n=20)$, and low $(n=55)$ prevalence (for Spain) Nocardia strains belonging to 30 species were identified via 16S rRNA and MALDI-TOF MS analysis. The manufacturer-provided database MALDI Biotyper library v4.0 (5.627 entries, Bruker Daltonik) was employed. In the high prevalence group (Nocardia farcinica, N. abscessus, $N$. cyriacigeorgica and $N$. nova), the $16 \mathrm{~S}$ rRNA and MALDI-TOF MS methods provided the same identification for $76 \%$ of the strains examined. For the intermediate prevalence group (N. brasiliensis, N. carnea, N. otitidiscaviarum and N. transvalensis complex), this figure fell to $45 \%$. In the low-prevalence group (22 species), these two methods were concordant only in six strains at the species level. Tetra-gene multi-locus sequencing analysis (MLSA) involving the concatemer gyrB-16S rRNA-hsp65-secA1 was used to arbitrate between discrepant identifications $(n=67)$. Overall, the MLSA confirmed the results provided at species level by $16 \mathrm{~S}$ rRNA analysis in 34.3\% of discrepancies, and those provided by MALDI-TOF MS in 13.4\%. MALDI-TOF MS could be a strong candidate for the identification of Nocardia species, but only if its reference spectrum database improves, especially with respect to unusual, recently described species and species included in the described Nocardia complexes.

Keywords: Nocardia spp., MALDI-TOF MS, 16S rRNA identification, MLSA, prevalence in Spain

\section{INTRODUCTION}

The members of Nocardia are branched Gram-positive opportunistic pathogens that live in soils rich in organic matter. Nearly 90 species (Euzeby, 2013) cause clinical problems, including cutaneous respiratory and systemic disease. Over $85 \%$ of all Nocardia species were identified during the molecular era; previously, time-consuming phenotypic tests were used (Brown-Elliott et al., 2015). Nowadays, the sequencing of the 16S rRNA gene is the most 
widespread method (Brown-Elliott et al., 2015). However, other techniques based on protein profiling, such as matrix-assisted laser desorption ionization-time of flight mass spectrometry (MALDI-TOF MS), have increased their throughput and potential use (Singhal et al., 2015) and have been shown useful in the routine laboratory identification of Nocardia (Carbonnelle et al., 2010).

Unfortunately, the MALDI-TOF MS identification harbors limitations when only manufacturer-provided database has been applied. It was stated in a literature review displaying low values of correct Nocardia species assignment $(\approx 15-53 \%$ ) (Xiao et al., 2016). In addition, the constantly increasing number of, recognized Nocardia species means that the commercial MALDITOF MS spectrum database for the genus can become outdated. Both facts made that requiring laboratories to compile their own libraries to get a successful identification (Brown-Elliott et al., 2015; Buckwalter et al., 2016). However, this step could not be feasible for many of the routine clinical laboratories, in which MALDI-TOF MS has been widely introduced.

To achieve a successful species assignment in this genus, molecular techniques as full-length 16S rRNA gene or multi-locus sequence analysis (MLSA) have been undergone. Both are high cost techniques that need on-site sequencing facilities, not being available in most of the clinical laboratories (Xiao et al., 2016).

The present work compares the agreement between 16S rRNA full gene and MALDI-TOF MS identification of Nocardia species by using the current commercial database without in-house supplementation (MALDI Biotyper software package version 3.1) and identifies the shortcomings of the latter. This analysis has been performed in a wide population of clinical Nocardia strains constituted by species with different prevalence in Spain. The tetra-gene MLSA (McTaggart et al., 2010) involving the concatemer gyrB-16S rRNA-hsp65-secA1 was used to arbitrate between discrepant identifications.

\section{MATERIALS AND METHODS}

\section{Bacterial Strains, Amplifications, and Sequencing}

The bacteria examined in this work were 100 Nocardia strains recovered from clinical samples (84 of respiratory origin, 6 cutaneous, 2 from the central nervous system and 8 from other tissues) sent to our reference centre (Spanish National Centre for Microbiology) from different hospitals between 2006 and 2014. These were identified by 16S rRNA analysis (see below) as representing 30 Nocardia species, and were selected for the present study since they represented more and less commonly encountered members of the genus. All these bacteria were incubated on buffered charcoal yeast extract agar (BCYE) or Columbia $5 \%$ sheep blood agar at $37^{\circ} \mathrm{C}$ for at least $48 \mathrm{~h}$ (i.e., until growth was clearly visible). DNA was extracted by the boiling method and amplifications of the studied genes were performed using Ready-To-Go PCR Beads (Amersham Biosciences, Buchinghamshire, UK). The products were electrophoresed and purified using Exo SAPITTM reagent (GE Healthcare, NJ, USA), and sequenced by capillary electrophoresis in a ABI PRISM 3100 apparatus (Applied Biosystems, Foster City, CA, USA) (Carrasco et al., 2013) PCR primers and conditions are listed in Supplementary Table S1.

\section{S rRNA Analysis}

The received Nocardia strains were identified at the species level by sequencing of the full-length $16 \mathrm{~S}$ rRNA gene ( size $\sim 1215 \mathrm{bp}$ ) (with proofreading and editing as necessary). The obtained sequences were compared with those in the GenBank database; those showing $\geq 99.6 \%$ similarity were deemed to be the same and thus positively identified following CLSI MM18 guidelines (Petti et al., 2008).

The BioEdit (Hall, 1999), CLUSTAL W (Thompson et al., 1994), and Mega 6.0 computer programs were used to construct a 16S rRNA phylogenetic tree (Tamura et al., 2013), employing the neighbor joining method (Gascuel, 1997) and Kimura 2 parameter distances (Kimura, 1980) (Figure 1). The reliability of the topologies was assessed by the bootstrap method with 1000 replicates.

Three groups were established according to the prevalence of Nocardia species in Spain (Carrasco et al., 2013): 25 strains representing Nocardia abscessus, $N$. cyriacigeorgica, $N$. farcinica and $N$. nova were assigned to the high prevalence group, 20 strains representing $N$. brasiliensis, $N$. carnea, $N$. otitidiscaviarum, and the $N$. transvalensis complex were assigned to the intermediate prevalence group, and 55 (representing 22 less frequently detected species) to the low prevalence group.

\section{MALDI-TOF MS Identification}

Protein samples were initially prepared as previously described (Verroken et al., 2010). As seen with Mycobacterium spp., the analysis of Nocardia by MALDI-TOF MS requires somewhat complicated sample preparation (Dunne et al., 2014) due to the presence of aliphatic acids in the cell wall (Xiao et al., 2016). Despite this fact, three modifications of this extraction technique were used (see Supplementary File S1): (i) mechanical disruption with glass-beads; (ii) 10 min of sonication at 2500 oscillation min-1 using a Mini Bead Beater (Biospec Products, Bartlesville, $\mathrm{OK}$ ) (both of these options preceding the rest of the Verroken et al. method); and (iii) freezing for $24 \mathrm{~h}$ before proceeding to protein extraction (performed after the boiling step described in the Verroken et al. method).

Matrix-assisted laser desorption ionization-time of flight spectra were randomly obtained from blind samples in the linear positive mode at a laser with a frequency of $20 \mathrm{~Hz}$ in the range of $2-20 \mathrm{kDa}$ with a Microflex $\mathrm{X}$ instrument using the FlexControl 3.3 (Bruker Daltonik, Bremen, Germany). Each recorded spectrum is the result of six series of 40 single laser shots in different locations, with user intervention only when needed. Raw spectra of bacteria were explored using the MALDI Biotyper 3.1 software package with default settings. The database for identification was the reference Biotyper library v4.0 5.627 MSP (Bruker Daltonik). In 2014, 5627 species were included in this database (Piau et al., 2015). Identification was performed with no custom database supplementation. Identification scores (MALDI-TOF MS log scores) of $\geq 2.0$ and 


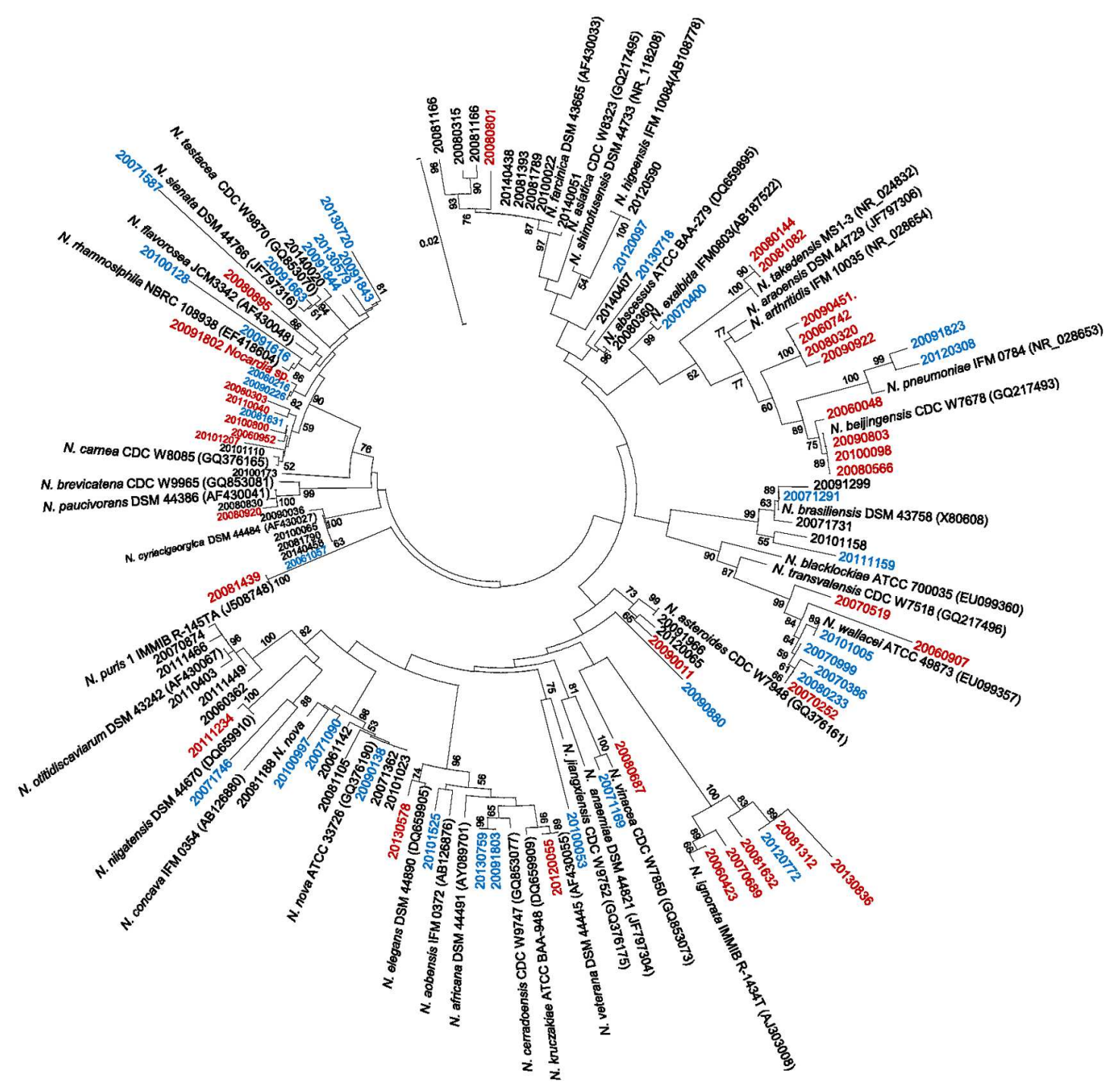

FIGURE 1 | Phylogenetic tree based on 16S rRNA analysis of 100 Nocardia spp. strains (collected in Spain between 2006 and 2014 ), plus type strains. The reliability of the topologies was assessed by the bootstrap method with 1000 replicates. The type strains, and the species for which full agreement was reached between the 16S rRNA and MALDI-TOF MS methods, are represented in black. Strains with partially concordant identifications are shown in blue. Strains for which no agreement was reached between the 16S rRNA and MALDI-TOF MS methods are shown in red.

$1.7-<2.0$ indicated species-level and genus-level identifications, respectively, (Verroken et al., 2010; Hsueh et al., 2014).

\section{Multi-Locus Sequence Analysis; Arbitration between Discrepant Identifications}

Multi-locus sequencing analysis was performed when the identifications made by the $16 \mathrm{~S}$ rRNA and MALDI-TOF MS methods did not agree $(n=67)$. The Nocardia identification pattern of McTaggart et al. (2010) was used, but with four genes instead of five; $16 \mathrm{~S}$ rRNA, gyrB, hsp65, and $\sec A 1$ genes. $r p o B$ was omitted given the reliability of this four-locus technique compared to the five-locus technique (99.5\%) (Liu et al., 2011). Concatenated sequences of these four genes from each discrepantly identified sample were aligned and a phylogenetic tree constructed. When type strains were not available from GenBank, those used in the McTaggart et al. study were employed (McTaggart et al., 2010). N. elegans, N. takedensis, and $N$. jiangxiensis lack a reference strain for all the genes in the MLSA pattern; they do not appear, therefore, in the phylogenetic tree. The results obtained were then compared against the discrepant identifications made by the $16 \mathrm{~S}$ rRNA and MALDI-TOF MS methods.

\section{Nucleotide Sequence Accession Numbers}

GenBank accession numbers for the sequences reported in this study are KT933407-KT933625. 
TABLE 1 | Level of agreement between 16S rRNA and commercial MALDI-TOF MS database (Bruker Biotyper library v.4.0) identification of Nocardia species.

\begin{tabular}{|c|c|c|}
\hline Nocardia spp. (no. of strains) & $\begin{array}{l}\text { Agreement between } 16 S \text { and } \\
\text { MALDI-TOF MS }\end{array}$ & $\begin{array}{c}\text { No. of strains in the Bruker } \\
\text { database }\end{array}$ \\
\hline \multicolumn{3}{|l|}{ High prevalence $(n=25)$} \\
\hline Nocardia abscessus $(n=2)$ & $2 / 2$ & 2 \\
\hline N. cyriacigeorgica $(n=6)$ & $5 / 6$ & 16 \\
\hline N. farcinica $(n=8)$ & $7 / 8$ & 12 \\
\hline N. nova $(n=9)$ & $5 / 9$ & 2 \\
\hline Total (\%) & $19 / 25(76.0 \%)$ & 32 \\
\hline \multicolumn{3}{|l|}{ Intermediate prevalence $(n=20)$} \\
\hline N. brasiliensis $(n=5)$ & $3 / 5$ & 1 \\
\hline N. carnea $(n=7)$ & $1 / 7$ & 1 \\
\hline N. otitidiscaviarum $(n=5)$ & $5 / 5$ & 6 \\
\hline N. transvalensis complex $(n=3)$ & $0 / 3$ & 1 \\
\hline Total $(\%)$ & $9 / 20(45 \%)$ & 9 \\
\hline \multicolumn{3}{|l|}{ Low prevalence $(n=55)$} \\
\hline 22 Nocardia species: & $5 / 55$ & 45 \\
\hline $\begin{array}{l}\text {-13 Nocardia species included in Bruker } \\
\text { database }(n=28)\end{array}$ & $5 / 23$ & 45 \\
\hline $\begin{array}{l}\text {-9 Nocardia species not included in Bruker } \\
\text { database }(n=27)\end{array}$ & 0 & 0 \\
\hline Total (\%) & $5 / 55(9.1 \%)$ & 45 \\
\hline
\end{tabular}

\section{RESULTS}

\section{Identification of Nocardia Species via MALDI-TOF MS, Resolving Discrepancies by MLSA}

Table 1 shows the degree of overall agreement between 16S and MALDI-TOF MS identification with manufacturer-provided database (MALDI Biotyper library v4.0), and that within each prevalence group according to its frequency in Spain (Carrasco et al., 2013). The 16S rRNA method identified 79 out of 100 studied strains at the species level (Petti et al., 2008); 13 could only be identified as Nocardia spp. Similarity values of 99.099.5\% were obtained for eight strains, not enough to assign them to any Nocardia species, although they can be assumed to belong to different Nocardia complexes (Schlaberg et al., 2013).

For the 33 strains with concordant $16 \mathrm{~S}$ rRNA and MALDI-TOF MS identifications at species level, the percentage similarity of the MLSA concatemer for each strain with respect to the reference sequences in GenBank reached 93.8-98.7\%, 90.7-97.4\%, and 90.1-100\% for the high, intermediate and low-prevalence subgroups, respectively.

For the 67 strains, not concordant in the identification made by the 16S rRNA and MALDI-TOF MS methods, MLSA was performed. Among them, 34 strains were concordant in genus but discrepantly identified at the species level by the $16 \mathrm{~S}$ rRNA and MALDI-TOF MS. Their percentage similarities of the MLSA concatemer for each strain with respect to the reference sequences for high, intermediate and low-prevalence subgroups were of
94.4-98.9\%, 96.9-97.6\%, and 94.4-100.0\%, respectively. MLSA confirmed 7 identifications made by $16 \mathrm{~S}$ rRNA and 9 identifications made by MALDI-TOF MS (Supplementary Table S2).

Further 33 strains -25 from the low prevalent groupwere not concordant even at genus level by $16 \mathrm{~S}$ rRNA and MALDI-TOF MS. MLSA confirmed 16 identifications made by $16 \mathrm{~S}$ rRNA and none identification was made by MALDI-TOF MS, as is shown in Supplementary Table S2. Their percentage similarities of the MLSA concatemer for each strain with respect to the reference sequences for high, intermediate and low-prevalence subgroups were of $99.8 \%$ (there was one strain only), 91.2-96.1 and 94.7-99.4\%, respectively (Supplementary Table S2). N. ignorata was excluded from analysis given the strong presence of degenerate positions in the reference strain partial 16S rRNA gene (N. ignorata IMMIB R-1434T; GenBank accession no. AJ303008).

\section{Identification of Nocardia High Prevalence Group}

With respect to the high prevalence group, agreement between the 16S rRNA and MALDI-TOF MS methods was reached for 19 of the 25 strains examined. However, agreement was complete only for the two strains of $N$. abscessus. One of the six discrepant strains (identified as $N$. farcinica by $16 \mathrm{~S}$ rRNA) was not identified even at genus level by the MALDI-TOF but laid together with its respective type strain in the MLSA tree. Among the five remaining strains, only one identification was confirmed by MLSA (Figure 2). 


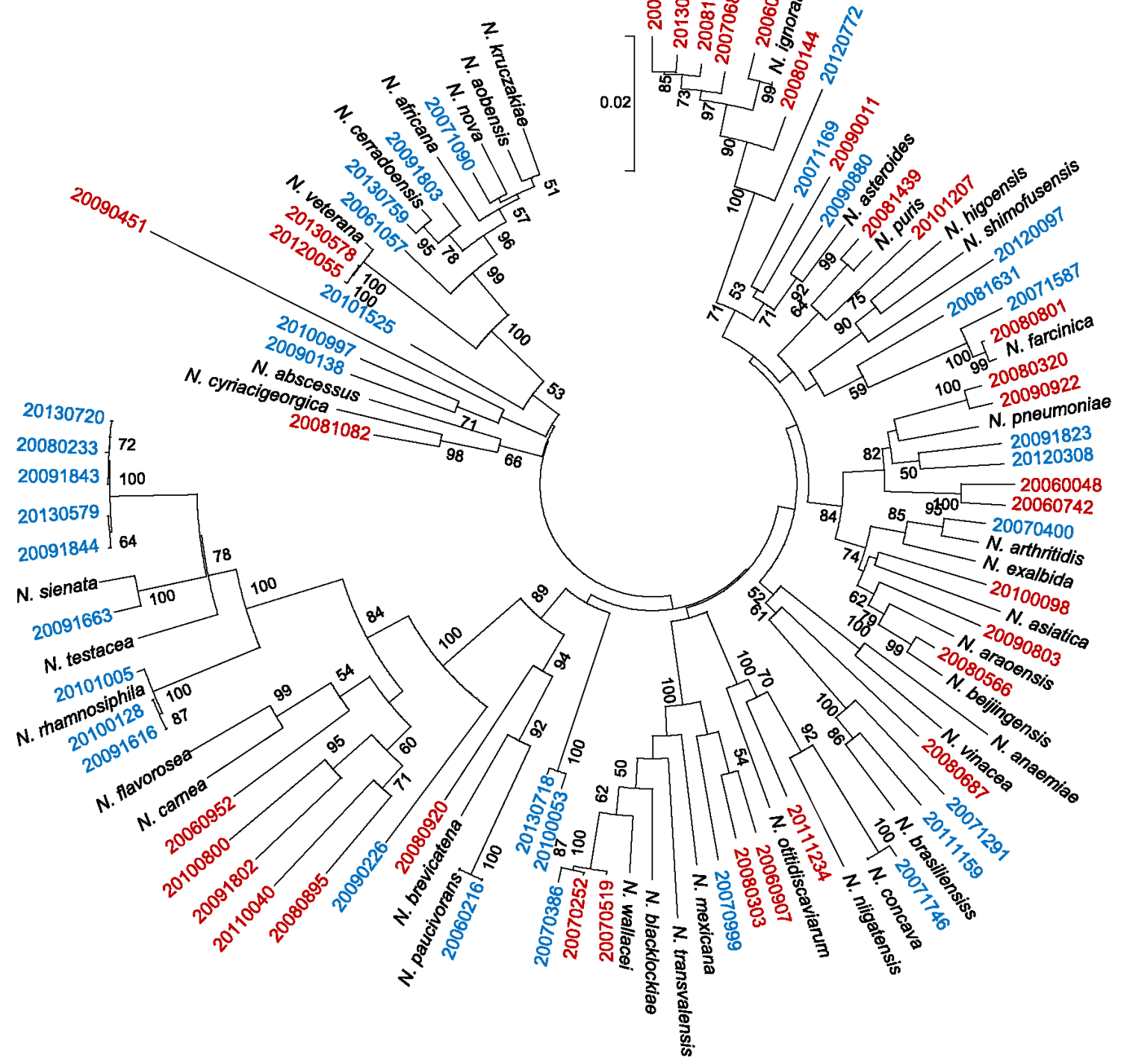

FIGURE 2 | Multi-locus sequencing analysis (MLSA)-based phylogenetic tree for 67 Nocardia spp. for which 16S rRNA and MALDI-TOF MS identification was discordant, plus type strains. The reliability of the topologies was assessed by the bootstrap method with 1000 replicates. The type strains are shown in black, and strains with partially concordant identifications are shown in blue. Strains for which no agreement was reached between the $16 \mathrm{~S}$ rRNA and MALDI-TOF MS methods are shown in red. Supplementary Table S2 contains further details.

\section{Identification of Nocardia Intermediate Prevalence Group}

The agreement between the $16 \mathrm{~S}$ rRNA and MALDITOF MS methods was reached for 9 of the 20 strains examined. However, while this agreement was complete only for the strains of $N$. otitidiscaviarum, no agreement was reached for any strain of the $N$. transvalensis complex. For the 11 strains with discrepant identification, good discrimination was reached by MLSA for $N$. brasiliensis, but not $N$. transvalensis complex. In addition, MLSA was unable to distinguish between $N$. carnea and $N$. flavorosea (Figure 2).

\section{Identification of Nocardia Low Prevalence Group}

16S rRNA and MALDI-TOF MS analysis only agreed in the species identification of just five strains in this group: 2 out of 4 examples of $N$. asteroides, 1 out of 1 example of $N$. higoensis, 1 out of 2 of $N$. paucivorans, and 1 out of $2 N$. testacea. For 25 of the total of 55 strains of this group, it was able to confirm their belonging to the genus Nocardia, but not for further 25 strains.

Only 13 of the 22 species in the low prevalence group include their mass spectra (MS) in the Bruker database $\mathrm{v}$ 4.0 (1-3 MS profiles per species), covering 23 out of 50 of the non-concordant strains of this group. With respect to 
the 13 species that were covered by the Bruker database, MLSA provided confirmation in the identifications at species level of 15 strains made by $16 \mathrm{~S}$ rRNA analysis (MALDITOF MS only provided identification for 4 strains). Indeed, for N. cerradoensis, N. ignorata, and N. pneumoniae, MLSA provided very good discrimination. MLSA was not able to confirm the $16 \mathrm{~S}$ rRNA identifications of $N$. wallacei or N. blacklockiae (MALDI-TOF MS only provided unreliable alternative identifications). MLSA tree was unable to distinguish between $N$. brevicatena/N. paucivorans, $N$. arthritidis/N. exalbida, and $N$. kruckzakiae/N. aobensis/N. africana/N. nova, as identified by $16 \mathrm{~S}$ rRNA (again, MALDI-TOF MS only provided unreliable alternative identifications with log scores <1.7).

Among the Nocardia strains belonging to non-covered species by MALDI-TOF v.4.0 library $(n=27)$, MLSA tree confirmed the identity of three strains - N. puris, N. rhamnosiphila, and $N$. shimofusensis - previously identified by the 16S rRNA method, but not by MALDI-TOF MS (Figure 2). No spectra were available in the Bruker database for $N$. beijingensis, N. flavorosea, and $N$. wallacei strains either, but these did locate to the same MLSA branch as their corresponding reference sequence, thus confirming to some degree their $16 \mathrm{~S}$ rRNA identifications. No MLSA confirmation could be made for the $N$. vinacea strains identified as such by $16 \mathrm{~S}$ rRNA analysis or as $N$. aobensis by MALDI-TOF MS. When no pattern for a Nocardia sp. type strain was available, only one strain returned a MALDI-TOF MS log score of $\geq 2.0$ allowing its identification at the species level. Two of the strains with discrepant identifications, but with MALDI-TOF MS log scores of 1.7-2.0, were identified by the latter as outside of the Nocardia genus. It is noteworthy that MALDI-TOF MS identified 11 Nocardia strains as Lactobacillus spp., one with a log score of 1.989 (Supplementary Table S2). However, four of the latter 11 strains also received an alternative identification as belonging to the genus Nocardia (log score range 1.16-1.42).

Of the 13 strains identified as belonging only to Nocardia sp. plus a type strain specification by the $16 \mathrm{~S}$ rRNA method (with 99.6\% similarity to their comparators in the GenBank database), although not identified as such by MALDI-TOF MS with the current commercial database, six were fully identified at the species level by the MLSA tree. Two strains with a $16 \mathrm{~S}$ rRNA-derived similarity of 99.0 and $99.5 \%$ to $N$. pneumoniae clustered in the MLSA phylogenetic tree with the N. pneumoniae DSM 44730 reference strain (GenBank accession no. JF797313). Besides, one Nocardia sp. strain unreliably identified by MALDI-TOF MS as $N$. paucivorans - but not identified as such by $16 \mathrm{~S}$ rRNA - clustered with $N$. paucivorans DSM 44386 (GenBank accession number AF430041) in the MLSA tree.

\section{DISCUSSION}

The MALDI-TOF MS method would appear to have a number of shortcomings with respect to the identification of Nocardia species. The Verroken et al. (2010) protein extraction protocol appears to be adequate but not for all the strains. When the unmodified method was used, 81 of the 100 strains were assigned an identification (though not necessarily correct). When those strains for which no identification was given were re-tested with either the additional glass bead, the sonication, or the $48 \mathrm{~h}$ freezing step, serviceable extracts were achieved for a further 10 , 5 , and 4 strains, respectively.

The agreement values reported by MALDI-TOF MS in an earlier study involving Gram-positive bacilli such as Lactobacillus spp., Listeria monocytogenes, and Rhodococcus spp. reached a much higher $\geq 80 \%$ (Farfour et al., 2012). However, less agreement was seen for 15 Kocuria (26.7\%) and 74 Nocardia strains (14.9\%) (Hsueh et al., 2014). Other authors have also indicated the inadequacy of the Bruker database for identifying Nocardia species, leading to identification agreements between the 16S rRNA and MALDI-TOF MS methods of just $15.6 \%$ (Segawa et al., 2015), 53.0\% (Khot et al., 2015), 42.0\% (Buckwalter et al., 2016), and none (Xiao et al., 2016) at the species level. However, these values were improved to 90.6, 83.1, 90.0 and $95.0 \%$, respectively, when in-house libraries with custom spectra were included.

The present results reveal the relatively poor agreement between the two methods in terms of Nocardia identification at the species level. While agreement was reached for $76 \%$ of the high-prevalence group species, this figure fell to 45 and $9.1 \%$, for those of the intermediate and low prevalence groups, respectively. The small number of MSs in the Bruker database for the high (especially for $N$. nova) and intermediate (especially for N. carnea and N. transvalensis complex) prevalence species, and for unusual and recently described species, renders the method unreliable because of the lack of identification by MALDI-TOF MS with the commercial v. 4.0 database. Moreover, in the present study, the identification of $>10 \%$ of the examined strains as members of Lactobacillus sp., as previously reported (Hsueh et al., 2014), is of particular concern.

When identifications were discrepant in Nocardia species, the MLSA method was used as an arbiter, to resolve the phylogenetic resolution at the species level (Glaeser and Kämpfer, 2015). When a discrepantly identified strain clustered with a type strain in the MLSA phylogenetic tree this allowed its final identification to be made (Vasileuskaya-Schulz et al., 2011). A problem with MLSA is the inexistence of identification breakpoints when using the present concatenated sequence. In addition, there is no consensus housekeeping gene system for use with Nocardia species, nor have codes been assigned to alleles, nor does any sequence database exist. Even so, it is the best method available for identifying Nocardia species, but much more expensive and laborious than 16S rRNA or MALDI-TOF MS. It is not, therefore, routinely used in health laboratories.

\section{CONCLUSION}

Until the Bruker database is amplified, the MALDI-TOF MS platforms with the Bruker Biotyper library v.4.0 cannot be 
considered a reliable technique as a routine method for resolving Nocardia sp. identification. It is specially meaningful for those unusual species -intermediate and low prevalence groups-, newly described and species included the Nocardia complexes. Until this occurs, health laboratories should consider retaining the use of the full-length $16 \mathrm{~S}$ rRNA gene sequencing and the reference laboratories should confirm by MLSA this identification.

\section{AUTHOR CONTRIBUTIONS}

GC contributed to the study design, the acquisition, analysis, and interpretation of data, and drafting the manuscript. SV, RC, and JS contributed to the study design, the interpretation of data and revising the manuscript. JC and NG contributed to the acquisition of data. All the authors have read and approved the final draft before submission.

\section{REFERENCES}

Brown-Elliott, B. A., Conville, P., and Wallace, R. J. (2015). Current status of Nocardia taxonomy and recommended identification methods. Clin. Microbiol. Newsl. 37, 25-32. doi: 10.1016/j.clinmicnews.2015.01.007

Buckwalter, S. P., Olson, S. L., Connelly, B. J., Lucas, B. C., Rodning, A. A., Walchak, R. C., et al. (2016). Evaluation of MALDI-TOF mass spectrometry for the identification of Mycobacterium species, Nocardia species and other aerobic actinomycetes. J. Clin. Microbiol. 54, 376-384. doi: 10.1128/JCM.02128-15

Carbonnelle, E., Mesquita, C., Bille, E., Day, N., Dauphin, B., Beretti, J. L., et al. (2010). MALDI-TOF MS mass spectrometry tools for bacterial identification in clinical microbiology laboratory. Clin. Biochem. 44, 104-109. doi: 10.1016/j.clinbiochem.2010.06.017

Carrasco, G., Valdezate, S., Garrido, N., Villalón, P., Medina-Pascual, M. J., and Sáez-Nieto, J. A. (2013). Identification, typing, and phylogenetic relationships of the main clinical Nocardia species in Spain according to their gyrB and $r p o B$ genes. J. Clin. Microbiol. 51, 3602-3608. doi: 10.1128/JCM.00515-13

Dunne, W. M. Jr., Doing, K., Miller, E., Miller, E., Moreno, E., Baghli, M. et al. (2014). Rapid inactivation of Mycobacterium and Nocardia species before identification using matrix-assisted laser desorption ionizationtime of flight mass spectrometry. J. Clin. Microbiol. 52, 3654-3659. doi: 10.1128/JCM.01728-14

Euzeby, J. P. (2013). List of Prokaryotic names with Standing in Nomenclature, 236. Available at: http://www.bacterio.net

Farfour, E., Leto, J., Barritault, M., Barberis, C., Meyer, J., Dauphin, B., et al. (2012). Evaluation of the Andromas matrix-assisted laser desorption ionization-time of flight mass spectrometry system for identification of aerobically growing Gram-positive bacilli. J. Clin. Microbiol. 50, 2702-2707. doi: 10.1128/JCM. 00368-12

Gascuel, O. (1997). BIONJ: an improved version of the NJ algorithm based on a simple model of sequence data. Mol. Biol. Evol. 14, 685-695. doi: 10.1093/oxfordjournals.molbev.a025808

Glaeser, S. P., and Kämpfer, P. (2015). Multilocus sequence analysis (MLSA). in prokaryotic taxonomy. Syst. Appl. Microbiol. 38, 237-245. doi: 10.1016/j.syapm.2015.03.007

Hall, T. A. (1999). BioEdit: a user-friendly biological sequence alignment editor and analysis program for Windows 95/98/NT. Nucleic Acids Symp. Ser. 41, 95-98.

Hsueh, P. R., Lee, T. F., Du, S. H., Teng, S. H., Liao, C. H., and Sheng, W. H. (2014). Bruker biotyper matrix-assisted laser desorption ionization-time of flight mass spectrometry system for identification of Nocardia, Rhodococcus, Kocuria, Gordonia, Tsukamurella, and Listeria species. J. Clin. Microbiol. 52, 2371-2379. doi: 10.1128/JCM.00456-14

\section{FUNDING}

This work was funded by a grant to NG from the Instituto de Salud Carlos III (MPY 1278/15).

\section{ACKNOWLEDGMENTS}

The authors are grateful to the CNM Biopolymers Unit for help in sequencing, to Adrian Burton for language and editing assistance, and to the health laboratories that provided the Nocardia strains examined.

\section{SUPPLEMENTARY MATERIAL}

The Supplementary Material for this article can be found online at: http://journal.frontiersin.org/article/10.3389/fmicb. 2016.00542

Khot, P. D., Bird, B., Durrant, R., and Fisher, M. A. (2015). Identification of Nocardia species by matrix-assisted laser desorption/ionization - time of flight mass spectrometry. J. Clin. Microbiol. 53, 3366-3369. doi: 10.1128/JCM.00780-15

Kimura, M. (1980). A Simple method for estimating evolutionary rate of base substitutions through comparative studies of nucleotide sequences. J. Mol. Evol. 16, 111-120. doi: 10.1007/BF01731581

Liu, W. L., Lai, C. C., Ko, W. C., Chen, Y. H., Tang, H. J., Huang, Y. L., et al. (2011). Clinical and microbiological characteristics of infections caused by various Nocardia species in Taiwan: a multicenter study from 1998 to 2010 . Eur. J. Clin. Microbiol. Infect. Dis. 30, 1341-1347. doi: 10.1007/s10096-0111227-9

McTaggart, L. R., Richardson, S. E., Witkowska, M., and Zhang, S. X. (2010). Phylogeny and identification of Nocardia species on the basis of multilocus sequence analysis. J. Clin. Microbiol. 48, 4525-4533. doi: 10.1128/JCM. 00883-10

Petti, C. A., Bosshard, P. P., Brandt, M. E., Clarridge, J. E., Feldblyum, T. V., Foxall, P., et al. (2008). Interpretive Criteria for Identification of Bacteria and Fungi by DNA Target Sequencing: Approved Guidelines, Vol. 28. Wayne, PA: Clinical and Laboratory Standards Institute.

Piau, C., Kerjouan, M., Le Mouel, M., Patrat-Delon, S., Henaux, P. L., Brun, V., et al. (2015). First case of disseminated infection with Nocardia cerradoensis in a human. J. Clin. Microbiol. 53, 1034-1037. doi: 10.1128/JCM. 02979-14

Schlaberg, R., Fisher, M. A., and Hanson, K. E. (2013). Susceptibility profiles of Nocardia strains based on current taxonomy. Antimicrob. Agents Chemother. 58, 795-800. doi: 10.1128/JCM.02979-14

Segawa, S., Nishimura, M., Sogawa, K., Tsuchida, S., Murata, S., Watanabe, M., et al. (2015). Identification of Nocardia species using matrix-assisted laser desorption/ionization-time-of-flight mass spectrometry. Clin. Proteomics 12, 1-8. doi: 10.1186/s12014-015-9078-5

Singhal, N., Kumar, M., Kanaujia, P. K., and Virdi, J. S. (2015). MALDITOF MS mass spectrometry: an emerging technology for microbial identification and diagnosis. Front. Microbiol. 6:791. doi: 10.3389/fmicb.2015. 00791

Tamura, K., Stecher, G., Peterson, D., Filipski, A., and Kumar, S. (2013). MEGA6: molecular evolutionary genetics analysis version 6.0. Mol. Biol. Evol. 30, 27252729. doi: $10.1093 / \mathrm{molbev} / \mathrm{mst} 197$

Thompson, J. D., Higgins, D. J., and Gibson, T. J. (1994). CLUSTAL $\mathrm{W}$ : improving the sensitivity of progressive multiple sequence alignment through sequence weighting, position-specific gap penalties and weight matrix choice. Nucleic Acids Res. 22, 4673-4680. doi: 10.1093/nar/22. 22.4673 
Vasileuskaya-Schulz, Z., Kaiser, S., Maier, T., Kostrzewa, M., and Jonas, D. (2011). Delineation of Stenotrophomonas spp. by multi-locus sequence analysis and MALDI-TOF MS mass spectrometry. Syst. Appl. Microbiol. 34, 35-39. doi: 10.1016/j.syapm.2010.11.011

Verroken, A., Janssens, M., Birhen, C., Bogaerts, P., Huang, T. D., Wauters, G., et al. (2010). Evaluation of matrix-assisted laser desorption ionization- time of flight mass spectrometry for identification of Nocardia species. J. Clin. Microbiol. 48, 4015-4021. doi: 10.1128/JCM. 01234-10

Xiao, M., Lu Pang, S. C., Fan, X., Zhang, L., Li, H. X., Hou, X., et al. (2016). Accurate identification of common pathogenic Nocardia species: evaluation of a multilocus sequence analysis platform and matrix-assisted laser desorption ionization-time of flight mass spectrometry. PLoS ONE 11:e0147487. doi: 10.1371/journal.pone.0147487
Conflict of Interest Statement: The authors declare that the research was conducted in the absence of any commercial or financial relationships that could be construed as a potential conflict of interest.

The reviewer MQ-B and handling Editor declared their shared affiliation, and the handling Editor states that the process nevertheless met the standards of a fair and objective review.

Copyright (๑) 2016 Carrasco, de Dios Caballero, Garrido, Valdezate, Cantón and Sáez-Nieto. This is an open-access article distributed under the terms of the Creative Commons Attribution License (CC BY). The use, distribution or reproduction in other forums is permitted, provided the original author(s) or licensor are credited and that the original publication in this journal is cited, in accordance with accepted academic practice. No use, distribution or reproduction is permitted which does not comply with these terms. 